\title{
Cyclin-dependent kinase-specific activity predicts the prognosis of stage I and stage II non-small cell lung cancer
}

\author{
Hiroshi Kubo ${ }^{*}$, Takashi Suzuki ${ }^{2}$, Tomoko Matsushima ${ }^{3}$, Hideki Ishihara ${ }^{3,7}$, Kazuya Uchino $^{4}$, Satoshi Suzuki ${ }^{5}$ \\ Sachiyo Tada ${ }^{3}$, Masahiro Yoshimura ${ }^{4}$ and Takashi Kondo ${ }^{6}$
}

\begin{abstract}
Background: Lung cancer is one of the leading causes of cancer death worldwide. Even with complete resection, the prognosis of early-stage non-small cell lung cancer is poor due to local and distant recurrence, and it remains unclear which biomarkers are clinically useful for predicting recurrence or for determining the efficacy of chemotherapy. Recently, several lines of evidence have indicated that the enzymatic activity of cyclin-dependent kinases could be a clinically relevant prognostic marker for some cancers. We investigated whether the specific activity of cyclin-dependent kinases 1 and 2 could predict recurrence or death in early non-small cell lung cancer patients.
\end{abstract}

Methods: Patients with newly diagnosed, pathologically confirmed non-small cell lung cancer were entered into this blinded cohort study. The activity of cyclin-dependent kinases was determined in 171 samples by the C2P® assay, and the results were subjected to statistical analysis with recurrence or death as a clinical outcome.

Results: The Cox proportional hazards model revealed that the activity of cyclin-dependent kinase 1, but not 2, was a predictor of recurrence, independent of sex, age, and stage. By contrast, cyclin-dependent kinase 2 activity was a predictor of death, independent of sex and stage.

Conclusion: This study suggested the possible clinical use of cyclin-dependent kinase 1 as a predictor of recurrence and cyclin-dependent kinase 2 as a predictor of overall survival in early-stage non-small cell lung cancer. Thus, a combination of activity of cyclin-dependent kinases 1 and 2 is useful in decision-making regarding treatment strategies for non-small cell lung cancer after surgery.

Keywords: Non-small cell lung cancer, Cyclin-dependent kinase, Surgical resection, Recurrence, Mortality

\section{Background}

Lung cancer is one of the leading causes of cancer death worldwide. Despite recent advances in cancer treatment, the prognosis of lung cancer is not sufficient compared with that of other solid organ tumors [1]. Even after complete surgical resection, the 5-year survival of earlystage non-small cell lung cancer (NSCLC) patients is only approximately $65 \%[2-4]$. This poor prognosis is due to the high recurrence after resection $[5,6]$, which supports the

\footnotetext{
* Correspondence: hkubo@med.tohoku.ac.jp

'Department of Advanced Preventive Medicine for Infectious Disease, Tohoku University Graduate School of Medicine, 2-1 Seiryoumachi, Aobaku, Sendai 980-8575, Japan

Full list of author information is available at the end of the article
}

presence of occult metastases. The survival benefit of adjuvant platinum-based chemotherapy has been established in stage II-III NSCLC [7-9]; however, there is no data supporting the use of adjuvant treatments for stage IA NSCLC, and the use of adjuvant chemotherapy for stage IB NSCLC is controversial [10]. Recently developed molecular biomarkers predict only non-squamous NSCLC $[11,12]$, and no biomarkers for squamous cell carcinoma (SCC) have reached the validation stage. Therefore, in the setting of lung cancer, the identification of biomarkers for predicting the outcome after surgery and selecting patients who could benefit from adjuvant chemotherapy is crucial.

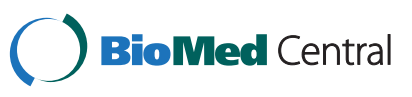

(c) 2014 Kubo et al.; licensee BioMed Central Ltd. This is an Open Access article distributed under the terms of the Creative Commons Attribution License (http://creativecommons.org/licenses/by/4.0), which permits unrestricted use, distribution, and reproduction in any medium, provided the original work is properly credited. The Creative Commons Public Domain Dedication waiver (http://creativecommons.org/publicdomain/zero/1.0/) applies to the data made available in this article, unless otherwise stated. 
A breakdown in the cell cycle machinery induces the uncontrolled proliferation of tumors. This process is initiated by a variety of molecules in a cascade that activates the cyclin-dependent kinases (CDKs), which play a role in the progression of the cell cycle. On the molecular level, the activity of CDKs is regulated by subunits known as cyclins, and by phosphorylation and dephosphorylation of key residues, for example, Thr14, Tyr15, and Thr160 in CDK2 [13]. A series of pathological investigations of the molecules that stimulate CDKs have clearly demonstrated their clinical significance for cancer diagnosis and treatment. For example, clinical evidence has indicated that the overexpression of cyclin $\mathrm{E}$ and cyclin $\mathrm{B}$, which bind to and activate $\mathrm{CDK} 2$ and $\mathrm{CDK} 1$, respectively, correlates with tumorigenesis, prognosis, and sensitivity to chemotherapy in a variety of malignancies, as does the inactivation of CDK inhibitors, such as p21WAF1 and p27Kip1 [14-20]. The pairing between the CDK and cyclin isotypes is specific. However, the amount of cyclin protein did not correspond perfectly with CDK activity in our investigation (data not shown). Similar results regarding the association between cyclin $\mathrm{E}$ and the activity of its associated kinase were reported by another group [21]. Therefore, we hypothesized that the direct measurement of CDK activity might produce relevant clinical indications for cancer diagnosis and treatment. Previously, we reported that the CDK-based risk score ( $\mathrm{C}_{2} \mathrm{P}^{\bullet}$ assay, Sysmex, Japan) predicted the risk of distant recurrence in early breast cancer patients [22]. The $\mathrm{C}_{2} \mathrm{P}^{\oplus}$ assay is determined using a combination of the specific activity of CDK1 (CDK1SA) and CDK2 (CDK2SA). The feasibility of this assay was confirmed in a cohort study in Caucasian breast cancer patients [23] and in colon cancer patients [24]. CDKSAs were also significantly associated with a pathologically complete response (pCR) after weekly administration of paclitaxel followed by 5-fluorouracil, epirubicin, and cyclophosphamide in breast cancer [25].

In lung cancer, many cell cycle-related molecules have been reported to be correlated with prognosis [26-31]. Here, we investigated whether the activity of CDK1 and CDK2 could predict the recurrence of NSCLC or the death of stage I and II NSCLC patients.

\section{Methods}

\section{Study design}

This blinded cohort study was approved by the local ethics committees of Tohoku University and Hyogo Cancer Centre. All patients provided written informed consent. A total of 213 patients who were newly diagnosed with pathologically confirmed NSCLC at the two centers were enrolled in this study. The eligibility criteria were as follows: SCC, adenocarcinoma, and stage I-II disease. All patients underwent complete resection, and none received adjuvant or neoadjuvant chemotherapy. CDK1SA and CDK2SA were determined in 171 samples using a $\mathrm{C}_{2} \mathrm{P}^{\oplus}$ assay (Sysmex, Kobe, Japan), and the results were subjected to statistical analysis to evaluate recurrence or death as a clinical outcome. Tumor tissue was dissected immediately after resection, snap-frozen and stored at $-80^{\circ} \mathrm{C}$ at each facility. Then, the samples were sent to the Sysmex Corporation (Kobe, Japan) and subjected to the $\mathrm{C}^{2} \mathrm{P}^{\circ}$ assay. Tissues with extreme blood contamination were excluded from this study, because the expression level of CDKs is underestimated in the presence of more than $1600 \mathrm{ng} / \mu \mathrm{L}$ of hemoglobin. The histologic types were centrally reviewed at Tohoku University.

\section{Patients}

A total of 213 patients with primary NSCLC who had undergone surgery between July 2000 and September 2009 were recruited for this study. Twenty-four cases were excluded due to extreme blood contamination of the samples. $\mathrm{C}_{2} \mathrm{P}^{\oplus}$ assay measurements were performed on 189 frozen samples; in 18 cases, the CDK expression levels were below the detection threshold, and these samples were excluded from the analysis. Finally, 171 cases were subjected to statistical analyses, including 53 SCCs and 118 adenocarcinomas. The median follow-up period was 43.9 months (70-2820 days).

\section{Measurement of CDK1SA and CDK2SA}

The $\mathrm{C}_{2} \mathrm{P}^{\oplus}$ assay $[15,22]$ was used to measure the specific activity of CDKs. In brief, lysates of freshly frozen samples were applied to the wells of a 96-well PVDF filter plate (Millipore, Billerica, MD, USA). The expression of CDK protein was detected quantitatively by sequential reactions with primary anti-CDK antibodies, biotinylated anti-rabbit antibodies and fluorescein-labeled streptavidin. To measure the kinase activity, CDK molecules were immunoprecipitated from the lysate using protein beads. CDK SA activity (maU/eU) was calculated as $\mathrm{CDK}$ activity units (maU/ $\mu \mathrm{L}$ lysate), which were divided by their corresponding CDK expression units (eU/ $\mu \mathrm{L}$ lysate). maU (CDK activity unit) and eU (CDK expression unit) were defined as the enzyme activity and expression equivalent to $1 \mathrm{ng}$ of recombinant kinase, respectively. When the expression level was lower than the detection limit of the assay, the case was excluded from the analysis. The detection limits for the expression level of CDK1 and CDK2 are 0.1 and 0.003 $\mathrm{eU} / \mu \mathrm{L}$ lysate, respectively.

\section{Statistical analysis}

Recurrence-free survival (RFS) was calculated from the date of surgery to the date of first local or distant recurrence; patients who were alive without recurrence at the time of data collection and those who died without any evidence of the disease on the date of death were censored. The overall 
survival (OS) was calculated from the date of surgery to the date of death; patients who were alive were censored.

The data were analyzed using MedCalc version 12.3 (MedCalc Software, Ostend, Belgium), and survival between the groups was compared using the Kaplan-Meier method and an unstratified Cox proportional hazards model or the log-rank test. Correlation tables were analyzed using the chi-square test. Receiver operating characteristic (ROC) curves and the corresponding area under the curve (AUC) for the compared models were computed to simulate predictive accuracy.

Possible prognostic variables that were analyzed in this study included age ( $\geq 70 v s<70$ years), sex (male or female), tumor size ( $>3 v s \leq 3 \mathrm{~cm}$ ), nodal status (negative or positive), pathological stage ( $\geq \mathrm{IB}$ vs IA), histological type (SCC or adenocarcinoma), CDK1SA $(\geq 12.6 v s<12.6)$ and CDK2SA ( $\geq 222 v s<222$ ). A value of $p<0.05$ was considered significant.

\section{Results}

We obtained fresh-frozen samples from 213 cases from two centers: Tohoku University Hospital and Hyogo Cancer Centre. Twenty-four cases were excluded due to extreme blood contamination. The $\mathrm{C}^{\circ} \mathrm{P}^{\circ}$ assay was performed on 189 frozen samples (see Additional file 1); in 18 cases, the CDK expression levels were below the detection threshold, and these cases were excluded from the analysis (assay success rate $=90 \%$ ). Finally, 171 samples were subjected to statistical analysis (Figure 1). The patients who were analyzed included 106 (62\%) males and 65 (38\%) females, with a median age of 70 years (38-86) (Table 1). The median tumor size was $3.0 \mathrm{~cm}(0.9-10.0)$. A total of 150 cases $(88 \%)$ were node-negative, and 21 cases $(12 \%)$ were positive. The histologic type was centrally confirmed as SCC in 53 cases (31\%) and adenocarcinoma in 118 cases (69\%). The overall recurrence rate and overall survival rate at final follow-up were $22 \%$ (local, $8 \%$; distant, 14\%) and 78\% (133/ 171), respectively. Thirty-five out of 37 recurrent cases received platinum-based chemotherapy. The distribution of CDK1SA and CDK2SA did not vary significantly between the two independent cohorts based on the chi-square test $(p=0.2102$ and $p=0.3557$, respectively; Table 2$)$. To examine the prognostic significance of the CDK1SA and CDK2SA results, ROC analysis was performed with overall recurrence as a clinical outcome.

The area under the ROC curves (AUC) of CDK1SA $(p=0.0498)$ and CDK2SA $(p=0.4206)$ were 0.607 and 0.545 , respectively, which indicated that CDK1SA, but not CDK2SA, was likely to be predictive of recurrence. The analysis revealed that the Youden Indexes for CDK1SA and CDK2SA were maximized at $12.6 \mathrm{maU} / \mathrm{eU}$ and $222 \mathrm{maU} / \mathrm{eU}$, respectively. Therefore, these values were tentatively set as the cut-off points in this study. Coincidentally, the optimal cut-off value of lung cancer approximated that of our colon study (11 maU/eU) [24]. The correlation analyses between CDKSA and the clinicopathologic parameters revealed that none of the parameters was associated with CDK1SA, while CDK2SA was significantly correlated with stage $(p=0.0267)$ and histology $(p<0.0001)$ (Table 2).

With a cut-off value of $12.6 \mathrm{maU} / \mathrm{eU}$, the cases were classified as low CDK1SA (54\%, 92 cases) or high CDK1SA (46\%, 79 cases). In the Kaplan-Meier analysis, patients with low CDK1SA tumors showed significantly higher RFS than those with high CDK1SA tumors based on a log-rank test (Figure 2A, HR 2.26, 95\% CI 1.184.32; $p=0.0147$ ); however, no prognostic value was observed (Figure 2B, $p=0.0921$ ).

CDK1SA and conventional clinicopathologic parameters, including sex (male vs female), age $(<70 v s \geq 70)$, tumor size $(\leq 3 \mathrm{~cm} v s>3 \mathrm{~cm})$, pathological lymph node status (positive $v s$ negative), clinical stage (IA $v s$ IB-IIB), and histology (SCC vs adenocarcinoma) were analyzed using a Cox proportional hazards model with recurrence or death as a clinical outcome (Tables 3 and 4). Univariate analysis for recurrence revealed that sex (HR 2.53, 95\% CI 1.16-5.52; $p=0.0200$ ), age (HR 2.80, 95\% CI $1.36-5.77 ; p=0.0054$ ), tumor size (HR 1.99, 95\% CI $1.04-3.81 ; p=0.0380$ ), pathological lymph node status (HR 3.20, 95\% CI 1.51-6.77; $p=0.0025$ ), stage (HR 2.54, 95\% CI 1.30-4.99; $p=0.0070$ ) and CDK1SA (HR 2.26, 95\% CI 1.16-4.43; $p=0.0177$ ) were statistically significant $(p<0.05)$. Age, stage and CDK1SA remained significant by multivariate analysis for recurrence (Table 3 , age

\section{3 primary stage I or II NSCLC patients underwent radical surgery without adjuvant chemotherapy between 2000 and 2009 \\ 42 patients 24 specimens with extreme blood contamination 18 specimens with no detectable CDK expression \\ 171 primary stage I or II NSCLC patients were analyzed in this study}

Figure 1 Flow chart of patient enrolment and reasons for exclusion. 
Table 1 Clinical and pathological characteristics of patients

\begin{tabular}{|c|c|c|c|c|}
\hline & & Total collective & Tohoku University & Hyogo Cancer Centre \\
\hline Frozen blocks available & & 213 & 111 & 102 \\
\hline Successful CDK assay & & 171 & 90 & 81 \\
\hline \multirow[t]{2}{*}{ Sex } & Male & $106(62 \%)$ & $57(63 \%)$ & $49(60 \%)$ \\
\hline & Female & $65(38 \%)$ & $33(37 \%)$ & $32(40 \%)$ \\
\hline \multirow[t]{2}{*}{ Age } & $<70$ years & $83(49 \%)$ & $43(48 \%)$ & 40 (49\%) \\
\hline & $\geq 70$ years & $88(52 \%)$ & $47(52 \%)$ & $41(51 \%)$ \\
\hline \multirow[t]{2}{*}{ Tumor size } & $\leq 3 \mathrm{~cm}$ & 95 (56\%) & $52(58 \%)$ & 43 (53\%) \\
\hline & $>3 \mathrm{~cm}$ & $76(44 \%)$ & $38(42 \%)$ & $38(47 \%)$ \\
\hline \multirow[t]{2}{*}{ pN } & - & $150(88 \%)$ & $83(92 \%)$ & $67(83 \%)$ \\
\hline & + & $21(12 \%)$ & $7(8 \%)$ & $14(17 \%)$ \\
\hline \multirow[t]{4}{*}{ Stage } & IA & $89(52 \%)$ & $48(53 \%)$ & $41(51 \%)$ \\
\hline & $\mathrm{IB}$ & $45(26 \%)$ & $28(31 \%)$ & $17(21 \%)$ \\
\hline & $\| \mathrm{A}$ & $31(18 \%)$ & $11(12 \%)$ & $20(25 \%)$ \\
\hline & $\| \mathrm{B}$ & $6(4 \%)$ & $3(3 \%)$ & $3(4 \%)$ \\
\hline \multirow[t]{2}{*}{ Histology } & Adenocarcinoma & $118(69 \%)$ & $58(64 \%)$ & $60(74 \%)$ \\
\hline & SCC & $53(31 \%)$ & $32(36 \%)$ & $21(26 \%)$ \\
\hline \multirow[t]{3}{*}{ Recurrence } & None & $134(78 \%)$ & $81(90 \%)$ & $53(65 \%)$ \\
\hline & Local & $14(8 \%)$ & $3(3 \%)$ & $11(14 \%)$ \\
\hline & Distant & $23(14 \%)$ & $6(7 \%)$ & $17(21 \%)$ \\
\hline \multirow[t]{2}{*}{ Survival information } & Alive & $133(78 \%)$ & 75 (83\%) & $58(72 \%)$ \\
\hline & Dead & 38 (22\%) & 15 (17\%) & 23 (28\%) \\
\hline
\end{tabular}

Table 2 Association between CDK-based risk groups and clinicopathological parameters

\begin{tabular}{|c|c|c|c|c|c|c|c|}
\hline & & \multicolumn{3}{|c|}{ CDK1SA (cut-off $=12.6$ ) } & \multicolumn{3}{|c|}{ CDK2SA (cut-off $=222$ ) } \\
\hline & & Low & High & $\overline{\text { Significance (Chi-square) }}$ & Low & High & Significance (Chi-square) \\
\hline \multirow[t]{2}{*}{ Sex } & Male & 57 & 49 & & 76 & 30 & \\
\hline & Female & 35 & 30 & $p=0.8817$ & 55 & 10 & $p=0.0800$ \\
\hline \multirow[t]{2}{*}{ Age } & $<70$ years & 43 & 40 & & 66 & 17 & \\
\hline & $\geq 70$ years & 49 & 39 & $p=0.7230$ & 65 & 23 & $p=0.4888$ \\
\hline \multirow[t]{2}{*}{ Tumor size } & $\leq 3 \mathrm{~cm}$ & 55 & 40 & & 76 & 19 & \\
\hline & $>3 \mathrm{~cm}$ & 37 & 39 & $p=0.2955$ & 55 & 21 & $p=0.3223$ \\
\hline \multirow[t]{2}{*}{$\mathrm{pN}$} & - & 85 & 65 & & 118 & 32 & \\
\hline & + & 7 & 14 & $p=0.0759$ & 13 & 8 & $p=0.1544$ \\
\hline \multirow[t]{4}{*}{ Stage } & IA & 53 & 36 & & 73 & 16 & \\
\hline & $\mathrm{IB}$ & 25 & 20 & & 37 & 8 & \\
\hline & $\| A$ & 12 & 19 & & 18 & 13 & \\
\hline & $\| B$ & 2 & 4 & $p=0.1652$ & 3 & 3 & $p=0.0267$ \\
\hline \multirow[t]{2}{*}{ Histology } & Adenocarcinoma & 64 & 54 & & 101 & 17 & \\
\hline & SCC & 28 & 25 & $p=0.9961$ & 30 & 23 & $p<0.0001$ \\
\hline \multirow[t]{2}{*}{ Facility } & Tohoku Univ & 53 & 37 & & 72 & 18 & \\
\hline & Hyogo CC & 39 & 42 & $p=0.2102$ & 59 & 22 & $p=0.3557$ \\
\hline
\end{tabular}



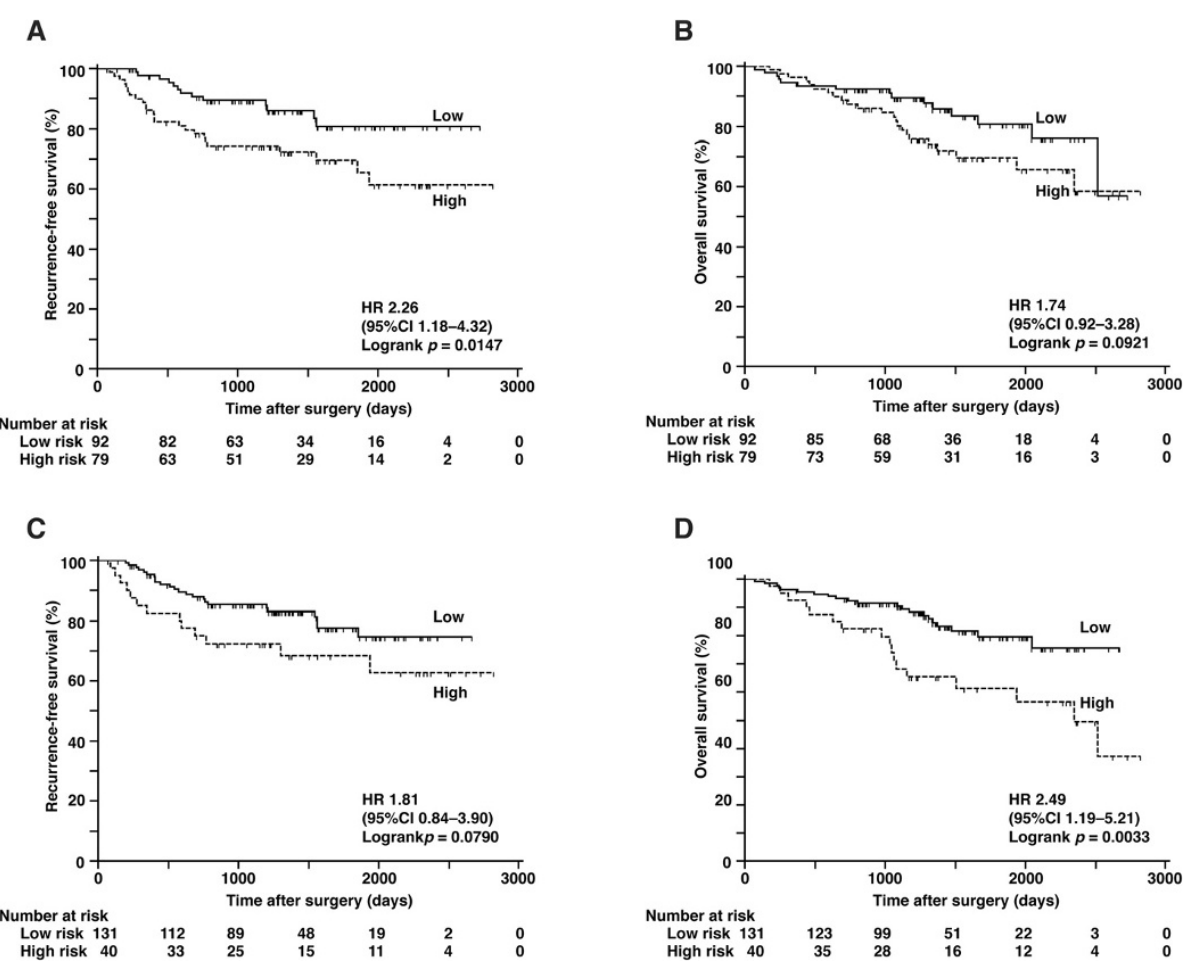

Figure 2 Analysis of recurrence and survival by risk category. (A) Recurrence-free survival and (B) overall survival according to CDK1SA-based risk with a cut-off value of $12.6 \mathrm{maU} / \mathrm{eU}$. (C) Recurrence- free survival and (D) overall survival according to CDK2SA-based risk with a cut-off value of $222 \mathrm{maU} / \mathrm{eU}$.

HR 3.06, $p=0.0028$; stage HR 2.16, $p=0.0306$; CDK1SA HR 2.25, $p=0.0195)$. Even in the subgroup of 134 patients with stage IA and IB disease, CDK1SA but not CDK2SA had the prognostic power (Figures $3 \mathrm{~A}$ and B, CDK1SA HR 2.56, $p=0.0273$; CDK2SA HR 1.18, $p=0.7375)$. The Cox regression analysis revealed that CDK1SA was an independent predictor of recurrence (Table 5, HR 2.57, 95\% CI 1.08-6.09; $p=0.0335$ ).

Regarding CDK2SA, which had a cut-off value of 222 $\mathrm{maU} / \mathrm{eU}$, the cases were classified as low CDK2SA (77\%, 131 cases) or high CDK2SA (23\%, 40 cases). Patients with low CDK2SA tumors showed significantly higher OS than those with high CDK2SA tumors based on a log-rank test (Figure 2D, HR 2.49, 95\% CI 1.19-5.21; $p=0.0033$ ).

According to the univariate analysis for death, CDK2SA (HR 2.56, 95\% CI 1.34-4.87; $p=0.0045$ ) was statistically significant along with sex (HR 5.51, 95\% CI $1.96-15.5 ; p=0.0013$ ), tumor size (HR 2.36, 95\% CI $1.22-4.55, p=0.0111$ ), pathological lymph node status (HR 2.82, 95\% CI 1.34-5.96; $p=0.0069)$ and stage (HR 2.75 , 95\% CI 1.39-5.44; $p=0.0039$ ). By multivariate analysis, sex, stage, and CDK2SA remained significant (sex HR 4.14, $p=0.0081$; stage HR 2.09, $p=0.0421$; CDK2SA HR 1.97, $p=0.0500$ ).

Table 3 Cox proportional hazards models for recurrence

\begin{tabular}{|c|c|c|c|c|c|c|c|}
\hline & & \multicolumn{2}{|c|}{ Univariate analysis } & \multicolumn{2}{|c|}{ Multivariate analysis } & \multicolumn{2}{|c|}{ Multivariate analysis } \\
\hline & & HR (95\% CI) & $p$ value & HR $(95 \% \mathrm{Cl})$ & $p$ value & HR $(95 \% \mathrm{Cl})$ & $p$ value \\
\hline Sex & Male & $2.53(1.16-5.52)$ & 0.0200 & $1.99(0.90-4.42)$ & 0.0913 & $2.00(0.91-4.40)$ & 0.0876 \\
\hline Age & $\geq 70$ years & $2.80(1.36-5.77)$ & 0.0054 & $3.06(1.48-6.34)$ & 0.0028 & $2.79(1.35-5.75)$ & 0.0056 \\
\hline Tumor size & $>3 \mathrm{~cm}$ & $1.99(1.04-3.81)$ & 0.0380 & & & & \\
\hline $\mathrm{pN}$ & + & $3.20(1.51-6.77)$ & 0.0025 & & & & \\
\hline Stage & $\geq \mathrm{IB}$ & $2.54(1.30-4.99)$ & 0.0070 & $2.16(1.08-4.31)$ & 0.0306 & $2.23(1.13-4.43)$ & 0.0222 \\
\hline Histology & $\mathrm{SCC}$ & $1.23(0.62-2.44)$ & 0.5567 & & & & \\
\hline CDK1SA & $\geq 12.6$ & $2.26(1.16-4.43)$ & 0.0177 & $2.25(1.14-4.42)$ & 0.0195 & & \\
\hline CDK2SA & $\geq 222$ & $1.82(0.93-3.56)$ & 0.0837 & & & $1.53(0.79-3.01)$ & 0.2165 \\
\hline
\end{tabular}


Table 4 Cox proportional hazards models for death

\begin{tabular}{|c|c|c|c|c|c|c|c|}
\hline & & \multicolumn{2}{|c|}{ Univariate analysis } & \multicolumn{2}{|c|}{ Multivariate analysis } & \multicolumn{2}{|c|}{ Multivariate analysis } \\
\hline & & HR $(95 \% \mathrm{Cl})$ & $p$ value & HR (95\% Cl) & $p$ value & HR $(95 \% \mathrm{Cl})$ & $p$ value \\
\hline Sex & Male & $5.51(1.96-15.46)$ & 0.0013 & $4.29(1.50-12.30)$ & 0.0070 & $4.14(1.45-11.78)$ & 0.0081 \\
\hline Age & $\geq 70$ years & $1.24(0.66-2.35)$ & 0.5088 & & & & \\
\hline Tumor size & $>3 \mathrm{~cm}$ & $2.36(1.22-4.55)$ & 0.0111 & & & & \\
\hline $\mathrm{pN}$ & + & $2.82(1.34-5.96)$ & 0.0069 & & & & \\
\hline Stage & $\geq \mathrm{IB}$ & $2.75(1.39-5.44)$ & 0.0039 & $1.79(0.87-3.71)$ & 0.1174 & $2.09(1.03-4.24)$ & 0.0421 \\
\hline Histology & SCC & $2.49(1.31-4.72)$ & 0.0053 & $1.53(0.78-2.97)$ & 0.2156 & $1.23(0.62-2.47)$ & 0.5555 \\
\hline CDK1SA & $\geq 12.6$ & $1.74(0.91-3.32)$ & 0.0962 & $1.53(0.79-2.97)$ & 0.2122 & & \\
\hline CDK2SA & $\geq 222$ & $2.56(1.34-4.87)$ & 0.0045 & & & $1.97(1.00-3.87)$ & 0.0500 \\
\hline
\end{tabular}

Subanalysis by histology revealed that the predictive value of CDK1SA for recurrence was stronger in adenocarcinoma than in SCC (Figure 4A and B, adenocarcinoma HR 2.26, $p=0.0439$; SCC HR 2.09, $p=0.2182$ ). On the contrary, in the SCC cases, the Cox regression analysis for recurrence revealed that only CDK2SA was statistically significant (Figure 4C and D, Table 6, HR 3.86, 95\% CI 1.05-14.2; $p=0.0428$ ).

In the distribution of 35 recurrent cases on a scatter diagram with logarithmic scales according to CDK1SA and CDK2SA, we observed that the distribution of the non-survivors slightly shifted to the higher CDKSAs area (data not shown). This observation let us to perform Kaplan-Meier analyses, and it was found that the prognostic power of CDK2SA, but not of CDK1SA (Figure 5A), was significant in 35 patients who were treated with chemotherapy after recurrence (Figure 5B, HR for death 4.30, 95\% CI 1.56-11.8; $p<0.0001)$.

\section{Discussion}

In this study, we demonstrated that CDK1SA and CDK2SA could identify individuals that were at high risk for recurrence and death among early-stage NSCLC patients after surgical resection. Even with complete resection, the prognosis of early-stage NSCLC is not good due to local and distant recurrence [6], and it remains unclear which biomarkers are clinically useful for predicting recurrence, although some single molecules and gene signatures of non-squamous cell carcinomas are being validated in a large number of cohorts [11,32]. Previously, cyclin expression and prognosis were reported to be correlated in lung cancer patients [33]; however, this is the first study revealing that CDK activity is a promising predictor for early-stage NSCLCs. Furthermore, CDK1SA and CDK2SA are the first biomarkers that can be used to predict the prognosis of both adenocarcinoma and SCC. Because CDKs are the targets of new anti-cancer drugs and the development of many CDK inhibitors is underway [34], this study will provide a basis for future personalized medicine using CDK inhibitors in NSCLC patients.

CDK1SA and CDK2SA have different implications in lung cancer. CDK1SA predicted recurrence after surgery, whereas CDK2SA predicted the overall survival of stage I and II NSCLC patients (Figure 2). Sub-analysis of 134 patients with stage IA and IB disease showed that CDK1SA was an independent predictor only for recurrence, but the power of prediction was much better than conventional criteria such as tumor size and stage (Figure 3 and Table 5). The clinical relevance of
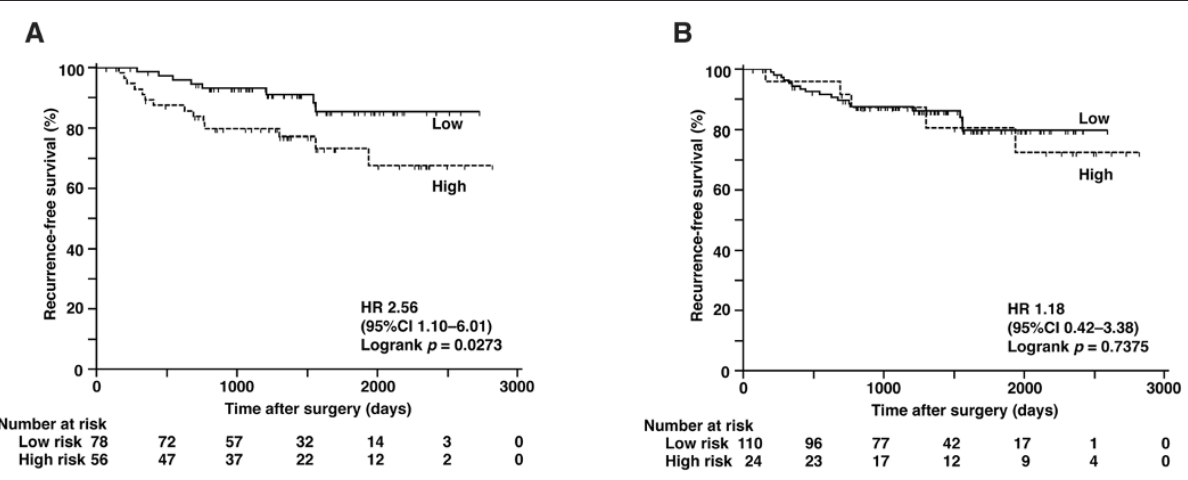

Figure 3 Analysis of recurrence by risk category in stage I NSCLC. (A) Recurrence-free survival according to CDK1SA-based risk with a cut-off value of $12.6 \mathrm{maU} / \mathrm{eU}$. (B) Recurrence-free survival according to CDK2SA-based risk with a cut-off value of $222 \mathrm{maU} / \mathrm{eU}$. 
Table 5 Cox proportional hazards models for recurrence (Stage I)

\begin{tabular}{lllc}
\hline & & \multicolumn{2}{c}{ Univariate analysis } \\
\cline { 3 - 4 } & & HR $(\mathbf{9 5 \%} \mathrm{Cl})$ & $\boldsymbol{p}$ value \\
\hline Sex & Male & $2.17(0.85-5.53)$ & 0.1050 \\
Age & $\geq 70$ years & $1.79(0.75-4.24)$ & 0.1908 \\
Tumor size & $>3 \mathrm{~cm}$ & $1.41(0.59-3.35)$ & 0.4413 \\
Stage & $\geq \mathrm{IB}$ & $1.67(0.71-3.90)$ & 0.2390 \\
Histology & SCC & $1.10(0.41-2.96)$ & 0.8568 \\
CDK1SA & $\geq 12.6$ & $2.57(1.08-6.09)$ & 0.0335 \\
CDK2SA & $\geq 222$ & $1.19(0.44-3.21)$ & 0.7381 \\
\hline
\end{tabular}

CDK1SA as a marker for recurrence prediction is in agreement with the conclusion of the colon study [24]. In this colon study, CDK1SA was significantly elevated in microsatellite-stable tumors. Because most of colorectal cancers with stable microsatellites demonstrate chromosomal instability [35], CDK1SA may have value as a marker of genomic instability. Genomic instability has been reported to predict clinical outcomes in multiple cancer types, including lung cancer [36-39]; therefore, prediction of recurrence demonstrated in this study may reflect the genomic instability of the tumors. According to sub-analysis by histology, the predictive value
Table 6 Cox proportional hazards models for recurrence (SCC)

\begin{tabular}{llcc}
\hline & & \multicolumn{2}{c}{ Univariate analysis } \\
\cline { 3 - 4 } & & HR $(\mathbf{9 5} \% \mathrm{Cl})$ & $\boldsymbol{p}$ value \\
\hline Sex & $\geq 70$ years & NA & \\
Age & $>3 \mathrm{~cm}$ & $1.48(0.47-4.65)$ & 0.5014 \\
Tumor size & + & $1.94(0.59-6.45)$ & 0.3905 \\
pN & $\geq$ IB & $2.42(0.54-10.99)$ & 0.2793 \\
Stage & $\geq 12.6$ & $2.09(0.63-6.91)$ & 0.2530 \\
CDK1SA & $\geq 222$ & $3.86(1.05-14.17)$ & 0.2286 \\
CDK2SA & &
\end{tabular}

NA, not analyzed due to bias ( $89 \%$ of SCC patients were male).

of CDK1SA for recurrence was greater in adenocarcinoma than in SCC (Figure 4A and B). Since adenocarcinoma is the most common histologic type of colon cancer, there is a similarity in the role of CDK1SA in colon and lung adenocarcinoma. Interestingly, Cox regression analysis for recurrence revealed that CDK2SA expression was statistically significant in SCC but not in adenocarcinoma (Figure 4C, D and Table 6). This result suggests that CDK1 and CDK2 have different roles in adenocarcinoma and SCC.

In contrast to CDK1SA, CDK2SA predicted overall survival after surgery. This finding may be related to the

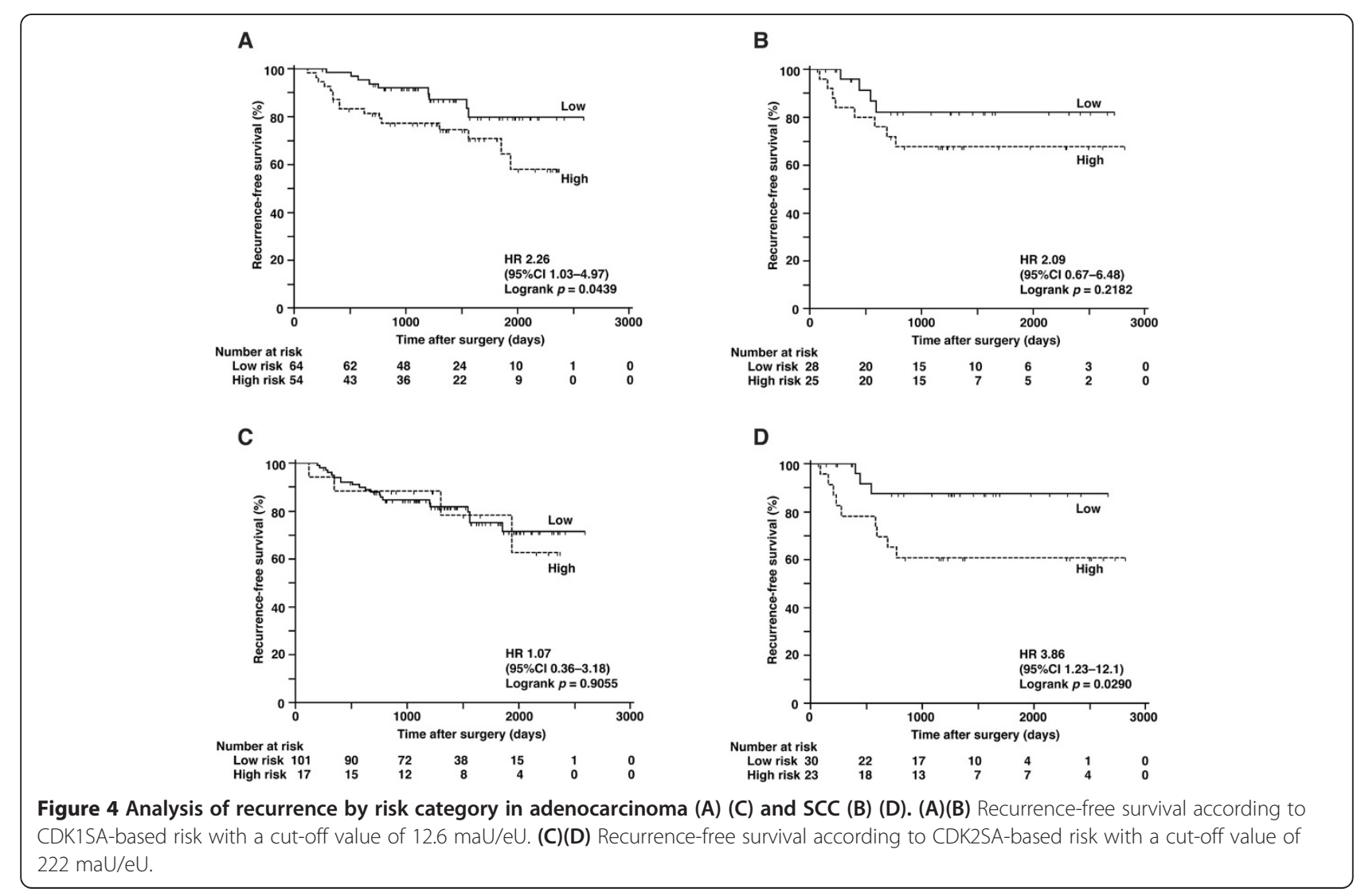



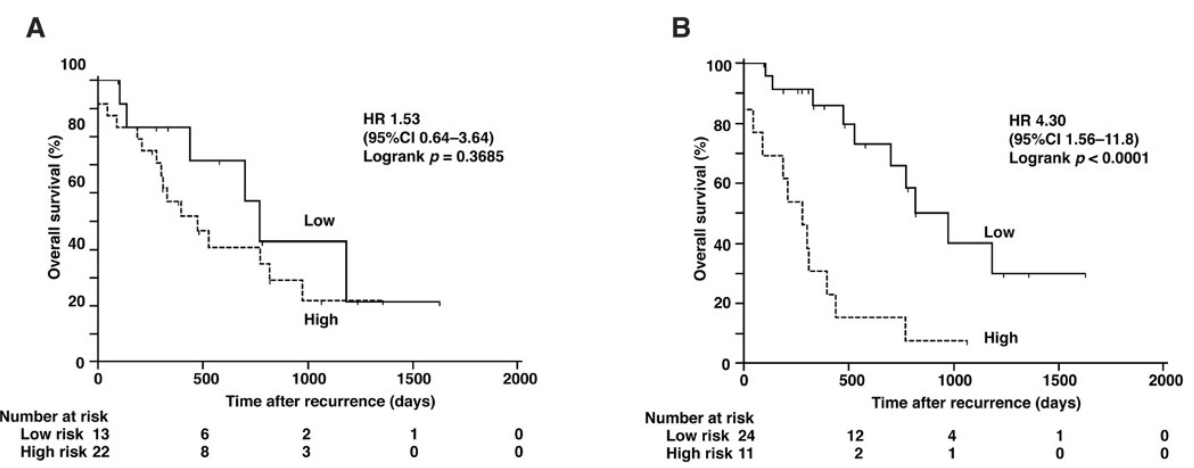

Figure 5 Analysis of survival by risk category in recurrent cases. The zero timepoint indicates the diagnosis of recurrence. (A) Survival according to CDK1SA-based risk with a cut-off value of $12.6 \mathrm{maU} / \mathrm{eU}$. (B) Survival according to CDK2SA-based risk with a cut-off value of $222 \mathrm{maU} / \mathrm{eU}$.

chemo-sensitivity of the patients. Kaplan-Meier analysis indicated that the prognostic power of CDK2SA was significant in patients treated primarily with platinumbased chemotherapy after recurrence (Figure 5), suggesting that CDK2SA can predict platinum sensitivity/ resistance. A similar trend was observed in our ovary study: tumors with high CDK2SA were more platinumresistant in patients who underwent incomplete resection and subsequent platinum-based chemotherapy (unpublished data). The ability of cyclin E-associated kinase activity to predict the response to platinum-based chemotherapy in ovarian cancer patients was also reported by another group [21]. In addition, inhibition of CDK2, but not CDK1, induced growth arrest in lung cancer cell lines through anaphase catastrophe [40]. Taken together, these data indicate that CDK2 would be a good target for lung cancer treatment, and the measurement of CDK2SA could be useful for identifying patients who would receive the full benefit of CDK2 inhibitors.

A limitation of this study was that the number of cases in the sub-analysis for the outcome after platinum-based chemotherapy was low; however, the significant difference was quite clear (Figure 5). Prospective studies should be performed to clarify the predictive capacity of CDK2SA in platinum sensitivity/ resistance in early-stage NSCLC patients after surgical resection.

In summary, this study suggested the possible clinical use of CDK1SA for recurrence prediction and CDK2SA for the prognosis of stage I and II NSCLC. Moreover, CDK2SA might be a predictor of platinum-based chemotherapy sensitivity/resistance. To the best of our knowledge, this is the first report that suggests a relationship between chemosensitivity and CDK activity in lung cancer. Thus, a combination of CDK1SA and CDK2SA might be helpful in decision-making regarding NSCLC treatment strategies.

\section{Conclusions}

CDK1SA is a predictor of recurrence and CDK2SA is a predictor of overall survival in early-stage NSCLC after surgery.

\section{Additional file}

Additional file 1: Distribution of lung tumors according to CDK1SA and CDK2SA. Adenocarcinoma cases and SCC cases are plotted on a scatter diagram with logarithmic scales according to CDK1SA and CDK2SA. Black square; the specific activity was defined as 0.5 when the activity of CDK is lower than the detection limit of the assay. The detection limits for the activity of CDK1 and CDK2 are 10 and $2 \mathrm{maU} / \mu \mathrm{L}$ lysate, respectively.

\section{Abbreviations}

NSCLC: Non-small cell lung cancer; SCC: Squamous cell carcinoma; CDKs: Cyclin-dependent kinases; CDK1SA: Specific activity of CDK1; CDK2SA: Specific activity of CDK2.

\section{Competing interests}

This study was supported by the Sysmex Corporation (Kobe, Japan). The sponsor was involved in the study design as well as the data collection, analysis, and interpretation. ST and TM of Sysmex Corporation had access to the full raw data. HI was previously affilaited with the Sysmex Corporation, was authorized to access to the primary raw data, and performed the initial analysis of this study. However, $\mathrm{HI}$ is now affilaited with the other company, Nittobo Medical Co. Ltd., which has no relation with the Sysmex Corporation; and has no access to the full raw data. Otherwise, the authors declare that they have no competing interests.

\section{Authors' contributions}

HK: Conception and design, collection and assembly of data, data analysis and interpretation, manuscript writing, final approval of manuscript; TS: Collection and assembly of data, pathological analysis and interpretation, final approval of manuscript; TM and ST: Collection and assembly of data, data analysis and interpretation, manuscript writing, final approval of manuscript; $\mathrm{HI}$ : Collection and assembly of data, data analysis and interpretation, final approval of manuscript; KU, SS, MY, and TK: Provision of study material and patients, obtain informed content from the patients, final approval of manuscript. All authors read and approved the final manuscript.

Acknowledgements

This work was supported by Sysmex Corporation, Kobe, Japan. 


\section{Author details}

${ }^{1}$ Department of Advanced Preventive Medicine for Infectious Disease, Tohoku University Graduate School of Medicine, 2-1 Seiryoumachi, Aobaku, Sendai 980-8575, Japan. ${ }^{2}$ Department of Pathology and Histotechnology, Tohoku University Graduate School of Medicine, 2-1 Seiryoumachi, Aobaku, Sendai 980-8575, Japan. ${ }^{3}$ Central Research Laboratories, Sysmex Corporation, 4-4-4, Takatsukadai, Nishi-ku, Kobe 651-2271, Japan. ${ }^{4}$ Department of General Thoracic Surgery, Hyogo Cancer Centre, 13-70 Kitaouji-chou Akashi 673-8558, Japan. ${ }^{5}$ Department of Thoracic Surgery, Japanese Red Cross Ishinomaki Hospital, 71 Nishimichishita, Hebita, Ishinomaki 986-8522, Japan. ${ }^{6}$ Department of Thoracic Surgery, Institute of Development, Aging and Cancer, Tohoku University, 4-1 Seiryoumachi, Aobaku, Sendai 980-8575, Japan. ${ }^{7}$ Present Address: R\&D Department, Nittobo Medical Co. Ltd., 1 Shiojima Fukuhara, Fukuyama, Koriyama 963-8061, Japan.

Received: 27 February 2014 Accepted: 3 October 2014 Published: 9 October 2014

\section{References}

1. Reck M, Heigener DF, Mok T, Soria JC, Rabe KF: Management of non-smallcell lung cancer: recent developments. Lancet 2013, 382:709-719.

2. Williams DE, Pairolero PC, Davis CS, Bernatz PE, Payne WS, Taylor WF, Uhlenhopp MA, Fontana RS: Survival of patients surgically treated for stage I lung cancer. J Thorac Cardiovasc Surg 1981, 82:70-76.

3. Nesbitt JC, Putnam JB Jr, Walsh GL, Roth JA, Mountain CF: Survival in earlystage non-small cell lung cancer. Ann Thorac Surg 1995, 60:466-472.

4. Spiro SG, Porter JC: Lung cancer-where are we today? Current advances in staging and nonsurgical treatment. Am J Respir Crit Care Med 2002, 166:1166-1196.

5. Martini N, Bains MS, Burt ME, Zakowski MF, McCormack P, Rusch WW, Ginsberg RJ: Incidence of local recurrence and second primary tumors in resected stage I lung cancer. J Thorac Cardiovasc Surg 1995, 109:120-129.

6. Kelsey CR, Marks LB, Hollis D, Hubbs JL, Ready NE, D'Amico TA, Boyd JA: Local recurrence after surgery for early stage lung cancer: an 11-year experience with 975 patients. Cancer 2009, 115:5218-5227.

7. Arriagada R, Bergman B, Dunant A, Le Chevalier T, Pignon JP, Vansteenkiste J, International Adjuvant Lung Cancer Trial Collaborative G: Cisplatin-based adjuvant chemotherapy in patients with completely resected non-smallcell lung cancer. N Engl J Med 2004, 350:351-360.

8. Douillard JY, Rosell R, De Lena M, Carpagnano F, Ramlau R, Gonzales-Larriba JL, Grodzki T, Pereira JR, Le Groumellec A, Lorusso V, Clary C, Torres AJ, Dahabreh J, Souquet PJ, Astudillo J, Fournel P, Artal-Cortes A, Jassem J, Koubkova L, His P, Riggi M, Hurteloup P: Adjuvant vinorelbine plus cisplatin versus observation in patients with completely resected stage IBIIIA non-small-cell lung cancer (Adjuvant Navelbine International Trialist Association [ANITA]): a randomised controlled trial. Lancet Oncol 2006, 7:719-727.

9. Pisters KM, Evans WK, Azzoli CG, Kris MG, Smith CA, Desch CE, Somerfield MR, Brouwers MC, Darling G, Ellis PM, Gaspar LE, Pass HI, Spigel DR, Strawn JR, Ung YC, Shepherd FA, Cancer Care Ontario, American Society of Clinical Oncology: Cancer Care Ontario and American Society of Clinical Oncology adjuvant chemotherapy and adjuvant radiation therapy for stages I-IIIA resectable non small-cell lung cancer guideline. J Clin Oncol 2007, 25:5506-5518

10. Strauss GM, Herndon JE 2nd, Maddaus MA, Johnstone DW, Johnson EA, Harpole DH, Gillenwater HH, Watson DM, Sugarbaker DJ, Schilsky RL, Vokes EE, Green MR: Adjuvant paclitaxel plus carboplatin compared with observation in stage IB non-small-cell lung cancer: CALGB 9633 with the Cancer and Leukemia Group B, Radiation Therapy Oncology Group, and North Central Cancer Treatment Group Study Groups. J Clin Oncol 2008, 26:5043-5051.

11. Kratz JR, He J, Van Den Eeden SK, Zhu ZH, Gao W, Pham PT, Mulvihill MS, Ziaei F, Zhang H, Su B, Zhi X, Quesenberry CP, Habel LA, Deng Q, Wang Z, Zhou J, Li H, Huang MC, Yeh CC, Segal MR, Ray MR, Jones KD, Raz DJ, Xu Z, Jahan TM, Berryman D, He B, Mann MJ, Jablons DM: A practical molecular assay to predict survival in resected non-squamous, non-small-cell lung cancer: development and international validation studies. Lancet 2012, 379:823-832.

12. Tomida S, Takeuchi T, Shimada Y, Arima C, Matsuo K, Mitsudomi T, Yatabe Y, Takahashi T: Relapse-related molecular signature in lung adenocarcinomas identifies patients with dismal prognosis. $J$ Clin Oncol 2009, 27:2793-2799.

13. Malumbres M, Barbacid M: Cell cycle, CDKs and cancer: a changing paradigm. Nat Rev Cancer 2009, 9:153-166.

14. Begnami MD, Fregnani JH, Nonogaki S, Soares FA: Evaluation of cell cycle protein expression in gastric cancer: cyclin B1 expression and its prognostic implication. Hum Pathol 2010, 41:1120-1127.

15. Ishihara H, Yoshida T, Kawasaki Y, Kobayashi H, Yamasaki M, Nakayama S, Miki E, Shohmi K, Matsushima T, Tada S, Torikoshi Y, Morita M, Tamura S, Hino Y, Kamiyama J, Sowa Y, Tsuchihashi Y, Yamagishi H, Sakai T: A new cancer diagnostic system based on a CDK profiling technology. Biochim Biophys Acta 2005, 1741:226-233.

16. Nakashima S, Natsugoe S, Matsumoto M, Kijima F, Takebayashi Y, Okumura H, Shimada M, Nakano S, Kusano C, Baba M, Takao S, Aikou T: Expression of p53 and p21 is useful for the prediction of preoperative chemotherapeutic effects in esophageal carcinoma. Anticancer Res 2000, 20:1933-1937.

17. Sjostrom J, Blomqvist C, Heikkila P, Boguslawski KV, Raisanen-Sokolowski A, Bengtsson NO, Mjaaland I, Malmstrom P, Ostenstadt B, Bergh J, Wist E, Valvere V, Saksela E: Predictive value of p53, mdm-2, p21, and mib-1 for chemotherapy response in advanced breast cancer. Clin Cancer Res 2000, 6:3103-3110.

18. Soria JC, Jang SJ, Khuri FR, Hassan K, Liu D, Hong WK, Mao L: Overexpression of cyclin B1 in early-stage non-small cell lung cancer and its clinical implication. Cancer Res 2000, 60:4000-4004.

19. Suzuki T, Urano T, Miki Y, Moriya T, Akahira J, Ishida T, Horie K, Inoue S, Sasano H: Nuclear cyclin B1 in human breast carcinoma as a potent prognostic factor. Cancer Sci 2007, 98:644-651.

20. Takano Y, Kato Y, van Diest PJ, Masuda M, Mitomi H, Okayasu I: Cyclin D2 overexpression and lack of p27 correlate positively and cyclin E inversely with a poor prognosis in gastric cancer cases. Am J Pathol 2000, 156:585-594.

21. Bedrosian I, Lee C, Tucker SL, Palla SL, Lu K, Keyomarsi K: Cyclin Eassociated kinase activity predicts response to platinum-based chemotherapy. Clin Cancer Res 2007, 13:4800-4806.

22. Kim SJ, Nakayama S, Miyoshi Y, Taguchi T, Tamaki Y, Matsushima T, Torikoshi Y, Tanaka S, Yoshida T, Ishihara H, Noguchi S: Determination of the specific activity of CDK1 and CDK2 as a novel prognostic indicator for early breast cancer. Ann Oncol 2008, 19:68-72.

23. van Nes JG, Smit VT, Putter H, Kuppen PJ, Kim SJ, Daito M, Ding J, Shibayama M, Numada S, Gohda K, Matsushima T, Ishihara H, Noguchi S, van de Velde CJ: Validation study of the prognostic value of cyclindependent kinase (CDK)-based risk in Caucasian breast cancer patients. Br J Cancer 2009, 100:494-500.

24. Zeestraten EC, Maak M, Shibayama M, Schuster T, Nitsche U, Matsushima T, Nakayama S, Gohda K, Friess H, van de Velde CJ, Ishihara H, Rosenberg R, Kuppen PJ, Janssen KP: Specific activity of cyclin-dependent kinase I is a new potential predictor of tumour recurrence in stage II colon cancer. Br J Cancer 2012, 106:133-140.

25. Kim SJ, Nakayama S, Shimazu K, Tamaki Y, Akazawa K, Tsukamoto F, Torikoshi Y, Matsushima T, Shibayama M, Ishihara H, Noguchi S: Recurrence risk score based on the specific activity of CDK1 and CDK2 predicts response to neoadjuvant paclitaxel followed by 5-fluorouracil, epirubicin and cyclophosphamide in breast cancers. Ann Oncol 2012, 23:891-897.

26. Dobashi $Y$, Jiang SX, Shoji M, Morinaga S, Kameya T: Diversity in expression and prognostic significance of $\mathrm{G} 1 / \mathrm{S}$ cyclins in human primary lung carcinomas. J Pathol 2003, 199:208-220.

27. Esposito V, Baldi A, Tonini G, Vincenzi B, Santini M, Ambrogi V, Mineo TC Persichetti P, Liuzzi G, Montesarchio V, Wolner E, Baldi F, Groeger AM: Analysis of cell cycle regulator proteins in non-small cell lung cancer. J Clin Pathol 2004, 57:58-63.

28. Hayashi H, Ogawa N, Ishiwa N, Yazawa T, Inayama Y, Ito T, Kitamura H: High cyclin E and low p27/Kip1 expressions are potentially poor prognostic factors in lung adenocarcinoma patients. Lung Cancer 2001, 34:59-65.

29. Jin $M$, Inoue S, Umemura T, Moriya J, Arakawa M, Nagashima K, Kato H: Cyclin D1, p16 and retinoblastoma gene product expression as a predictor for prognosis in non-small cell lung cancer at stages I and II. Lung Cancer 2001, 34:207-218.

30. Morero JL, Poleri C, Martin C, Van Kooten M, Chacon R, Rosenberg M: Influence of apoptosis and cell cycle regulator proteins on chemotherapy response and survival in stage IIIA/IIIB NSCLC patients. J Thorac Oncol 2007, 2:293-298. 
31. Yoshida T, Tanaka S, Mogi A, Shitara Y, Kuwano H: The clinical significance of Cyclin B1 and Wee1 expression in non-small-cell lung cancer. Ann Oncol 2004, 15:252-256.

32. Postel-Vinay S, Vanhecke E, Olaussen KA, Lord CJ, Ashworth A, Soria JC: The potential of exploiting DNA-repair defects for optimizing lung cancer treatment. Nat Rev Clin Oncol 2012, 9:144-155.

33. Singhal S, Vachani A, Antin-Ozerkis D, Kaiser LR, Albelda SM: Prognostic implications of cell cycle, apoptosis, and angiogenesis biomarkers in non-small cell lung cancer: a review. Clin Cancer Res 2005, 11:3974-3986.

34. Schwartz GK, Shah MA: Targeting the cell cycle: a new approach to cancer therapy. J Clin Oncol 2005, 23:9408-9421.

35. Walther A, Johnstone E, Swanton C, Midgley R, Tomlinson I, Kerr D: Genetic prognostic and predictive markers in colorectal cancer. Nat Rev Cancer 2009, 9:489-499.

36. Albertson DG, Collins C, McCormick F, Gray JW: Chromosome aberrations in solid tumors. Nat Genet 2003, 34:369-376.

37. Carter SL, Eklund AC, Kohane IS, Harris LN, Szallasi Z: A signature of chromosomal instability inferred from gene expression profiles predicts clinical outcome in multiple human cancers. Nat Genet 2006, 38:1043-1048

38. Mettu RK, Wan YW, Habermann JK, Ried T, Guo NL: A 12-gene genomic instability signature predicts clinical outcomes in multiple cancer types. Int J Biol Markers 2010, 25:219-228.

39. Nakamura H, Saji H, Idiris A, Kawasaki N, Hosaka M, Ogata A, Saijo T, Kato H: Chromosomal instability detected by fluorescence in situ hybridization in surgical specimens of non-small cell lung cancer is associated with poor survival. Clin Cancer Res 2003, 9:2294-2299.

40. Galimberti F, Thompson SL, Liu X, Li H, Memoli V, Green SR, DiRenzo J, Greninger P, Sharma SV, Settleman J, Compton DA, Dmitrovsky E: Targeting the cyclin E-Cdk-2 complex represses lung cancer growth by triggering anaphase catastrophe. Clin Cancer Res 2010, 16:109-120.

doi:10.1186/1471-2407-14-755

Cite this article as: Kubo et al:: Cyclin-dependent kinase-specific activity predicts the prognosis of stage I and stage II non-small cell lung cancer. BMC Cancer 2014 14:755.

\section{Submit your next manuscript to BioMed Central and take full advantage of:}

- Convenient online submission

- Thorough peer review

- No space constraints or color figure charges

- Immediate publication on acceptance

- Inclusion in PubMed, CAS, Scopus and Google Scholar

- Research which is freely available for redistribution 